\title{
Desenvolvimento de impressora 3D de coordenadas polares
}

\author{
Kalenin de Moraes Branco, Mariana Wong Yoshikawa
}

\begin{abstract}
Resumo - Com a crescente tendência de personalização de produtos de geometrias cada vez mais complexas, as impressoras 3D proporcionam uma grande versatilidade e têm sido utilizadas cada vez mais por diversos setores da sociedade, seja as de uso profissional por parte das empresas, quanto as de uso pessoal, pela comunidade em geral. Com um processo de fabricação chamado de tecnologia aditiva, as impressoras 3D tornam-se populares para diversos usuários. Com o intuito de tornar esta tecnologia mais acessível para a população, foi desenvolvida uma impressora 3D de coordenadas polares, com a vantagem de possuir uma quantidade menor de componentes que as impressoras 3D de coordenadas cartesianas.
\end{abstract}

Palavras-chave - Impressão 3D, RepRap, Coordenada Polar

\section{Introdução}

De acordo com Kristin Majcher (Majcher 2014), as empresas no mundo utilizam a manufatura aditiva principalmente para fazer protótipos para testes, porém nos últimos anos, este cenário mudou, e as empresas têm se utilizado das impressoras 3D para produzir peças e produtos funcionais. Com este aumento massivo de usuários de impressoras 3D, seja em âmbito profissional, com impressoras precisas e caras, ou em âmbito caseiro, com impressoras de qualidade inferior e baixo custo, se faz necessário o desenvolvimento de um novo tipo de impressora, que se utiliza de uma tecnologia já existente, mas que, movimentando-se em coordenadas polares, traz uma simplicidade maior em sua construção e um menor número de componentes, abaixando assim seu custo, e aumentando portanto sua acessibilidade.

O projeto proposto consiste no desenvolvimento de uma impressora 3D de FDM (Fused Deposition Modeling) com movimentação em coordenadas polares, diferente das usuais cartesianas. Com mecanismos mais simples e potencialmente mais leves, uma impressora 3D polar significaria um projeto mais simples e barato, sem perder qualidade de impressão.

Seguindo as diretrizes da filosofia RepRap (máquinas auto-replicáveis), foi utilizado o controlador Arduino Mega 2560, este com capacidade de controlar todos os componentes necessários. Em conjunto a esta placa é utilizado a RAMPS 1.4 para a integração. Há vários firmwares compatíveis com o Arduino Mega 2560, entre eles o Marlin360, é uma modificação do Marlin (firmware utilizado para coordenadas cartesianas) que faz a conversão para bases circulares, como máquinas Delta e Polares.

O mecanismo tipo bandeira, ou seja, um eixo horizontal translada sobre um eixo vertical ao contrário do mecanismo de pórtico, onde dois eixos verticais sustentam o eixo vertical, utiliza um número menor de componentes. Entretanto, com o aumento de suas dimensões, esta se torna instável com vibrações e deformações fora do requisito da máquina. Portanto, suas dimensões foram dimensionadas para atuar satisfatoriamente.

Com vários materiais disponíveis para tecnologia aditiva, o PLA se destaca por não necessitar ser depositado em uma base previamente aquecida, de modo a conferir sua aderência em relação ao ABS. Outro fator importante é a composição do PLA. Derivado do milho, este material é biodegradável. Uma característica que tem ganhado destaque diante da dificuldade de descarte de outros materiais plásticos.

Em vista dos argumentos apresentados, foi desenvolvida a impressora 3D com coordenadas polares. 


\section{Desenvolvimento}

\subsection{Metodologia}

O projeto está dividido em três etapas: o projeto da máquina, sua construção e avaliação através da impressão de peças, estas que serão utilizadas para a calibração da máquina. A primeira etapa, portanto, compreende o projeto mecânico da impressora $3 \mathrm{D}$ de coordenadas polares e a implementação da eletrônica e software. A seguir deve ser feita a montagem e teste, resultando então em peças impressas pela máquina.

\subsection{Requisitos de projeto}

Os requisitos para o projeto são constituídos pelos objetivos técnicos do projeto. Estes podem ser divididos em requisitos mecânicos, requisitos eletrônicos e requisitos de software.

\section{A Requisitos da mecânica}

- Ser constituído de 3 eixos de movimentação: eixo Z (altura), eixo R (raio) e eixo $\theta$ (ângulo).

- Possuir um sistema de extrusão e um sistema de aquecimento do filamento.

- Possuir um apoiador de filamento.

- Movimentação em vazio de $30 \mathrm{~m} / \mathrm{min}$.

- Aceleração de $5 \mathrm{~m} / \mathrm{s}^{2}$ para os eixos R e Z

- Aceleração linear de $2 \mathrm{~m} / \mathrm{s}^{2}$ para um raio de $5 \mathrm{~mm}$ no eixo $\theta$.

- Área útil de trabalho de $200 \mathrm{~mm}$ x $200 \mathrm{~mm}$ x $200 \mathrm{~mm}$

\section{B Requisitos da eletrônica}

- Fazer o processamento do movimento dos 3 eixos de locomoção

- Fazer o devido controle da extrusão do filamento

- Fazer o controle de temperatura do bico extrusor

- Controlar devidamente o sistema de resfriamento da máquina

\section{Requisitos de software}

- Controle manual da máquina

- Controle automático da máquina

- "Fatiamento" do modelo

- Módulo de importação de modelo em STL

- Geração do código G para enviar à máquina

\subsection{Software e Eletrônica}

O microcontrolador designado para a máquina foi o Arduino Mega 2560, este possui uma placa baseada no ATmega2560 (microcontrolador de 8-bit da Atmel com sistema de 256KB programável em Flash), possuindo 54 pinos de saídas e entradas digitais, dos quais quinze podem ser utilizadas como PWM - Pulse Width Modulation, uma técnica de se obter resultados analógicos por meios digitais (HIRZEL, 2015). A placa também possui um cristal oscilador de $16 \mathrm{MHz}$, conexão USB e botão de reset, portanto capaz de comportar todos elementos necessários para uma impressora e controla-los.

Foi decidido que será utilizado o RAMPS 1.4. RepRap Arduino Mega Pololu Shield (RAMPS), sendo este shield uma placa auxiliar estável para o Arduino Mega 2560, até 1/32 micropassos (obtido proporcionando a corrente de duas bobinas de modo a aumentar a resolução do motor de passo) e compatível com diversos firmwares amplamente utilizados, como Repetier-Firmware, Marlin e Sprinter (REPRAP, 2015). No caso da impressora 3D polar em questão, foi decido que o firmware deveria ter embutido um código de conversão de coordenadas cartesianas para coordenadas polares, para que pudesse ser utilizado um software e um fatiador (programa que gera o código G para uma fatia do sólido) amplamente utilizado. Infelizmente, após vários testes com 
diversos firmwares, houve diversos problemas na conversão para polar. Portanto foi utilizado o Repetier-Firmware, que faz o controle da temperatura, tradução do código $G$ em comando para motores, e a conversão foi feita manualmente.

O software escolhido para o processamento do modelo STL (STereoLithography, formato de arquivos para CAD criado pela 3D Systems) e ligação com o fatiador, além do IHM da máquina será o Repetier-Host (REPETIER, 2015) que é compatível com a maioria dos firmwares para RepRap utilizados. Este software possui a opção de escolher o fatiador (slicer) desejado para fatiar o arquivo STL, formando assim um conjunto de códigos $G$ para o comando da máquina, como por exemplo o "Slic3r". Vale lembrar que este código G gerado pelo fatiador é feito em coordenadas cartesianas e o firmware faz a conversão em coordenadas polares posteriormente. A solução final pode ser vista na Figura 1.

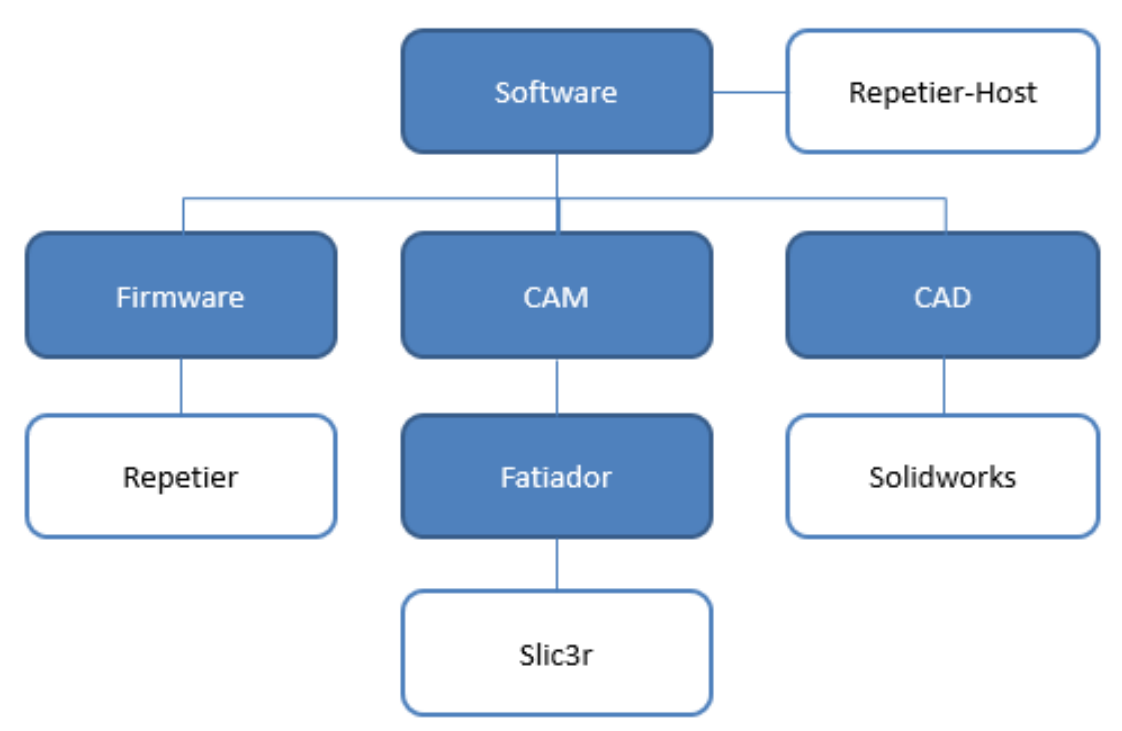

Figura 1 - Solução escolhida de Software. Fonte elaborada pelo autor.

\subsection{Estrutura mecânica}

Foram consideradas duas alternativas quanto a estrutura da impressora 3D. A primeira é em formato de portal como mostrado na Figura 2. Nesta alternativa, o bico extrusor está bi apoiado e translada em um eixo R. O eixo R se movimenta verticalmente através do eixo $\mathrm{Z}$, movido por dois motores de passo, um de cada lado da máquina. 


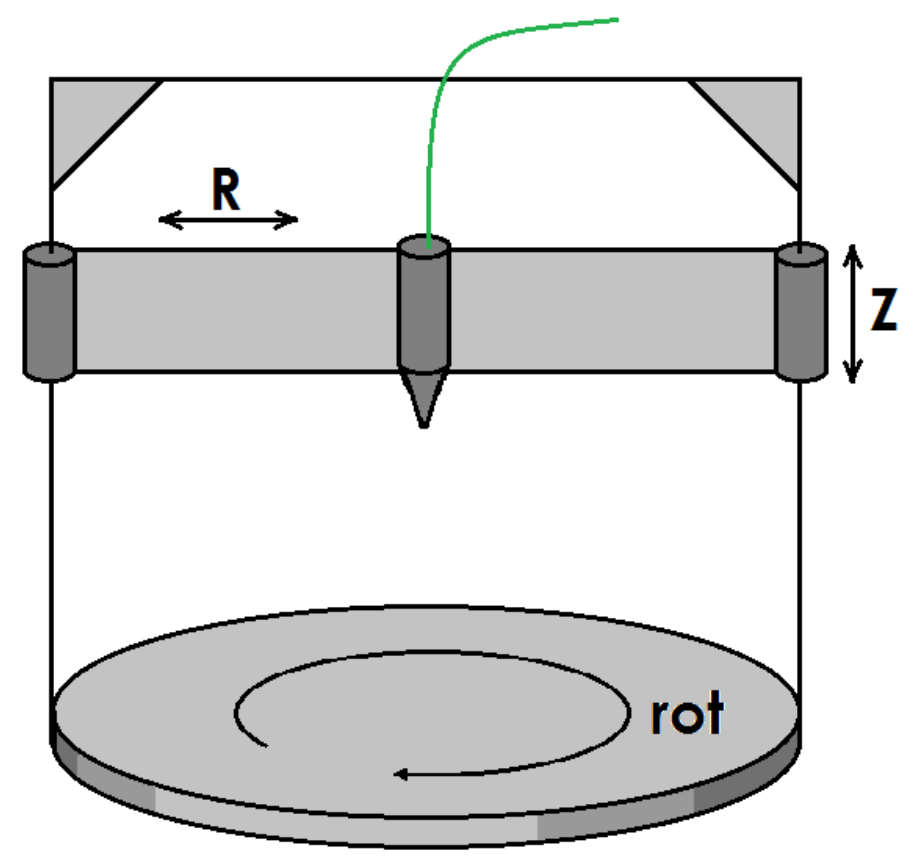

Figura 2 - Estrutura tipo portal. Fonte elaborada pelo autor.

A segunda opção trata-se da estrutura do tipo bandeira mostrada na Figura 3. Onde o bico extrusor translada no eixo $\mathrm{R}$ e este translada no eixo $\mathrm{Z}$ por apenas um motor de passo. Para esta configuração, a extrusora deve estar localizada fora da estrutura e ligada ao bocal através de um cabo. Essa modificação é feita para reduzir a massa movida do mecanismo, já que esta está em balanço.

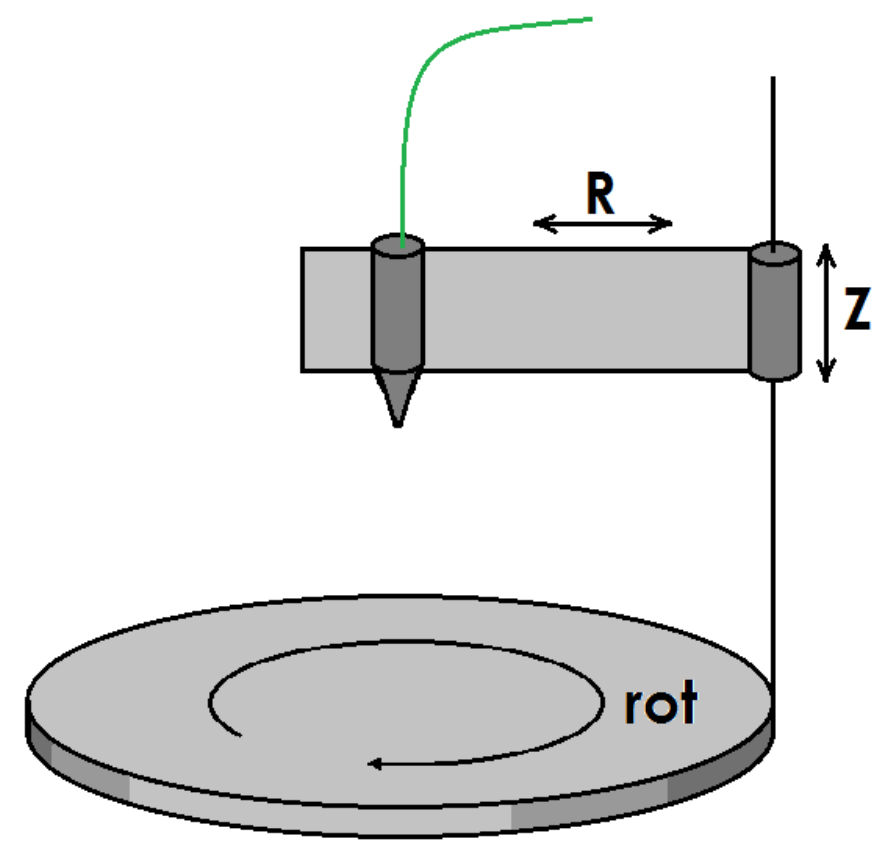

Figura 3 - Estrutura tipo bandeira. Fonte elaborada pelo autor.

\section{A Critérios de análise}

Para a análise da melhor solução a ser desenvolvida, foi usada uma matriz de decisão levando os seguintes critérios em consideração:

- Inércia Movida: Trata-se da massa total que é movida por um ou mais eixos de movimentação, quanto maior essa massa, pior é sua nota. Peso: 3

- Volume de Trabalho: Trata-se do volume útil de trabalho em comparação ao volume 
total da máquina. Quanto maior a máquina para um mesmo volume útil, pior é a nota. Peso: 2

- $\quad$ Custo: Neste quesito leva-se em conta a quantidade de peças envolvida no mecanismo. Quanto mais peças para um mecanismo, mais caro ele será e, portanto, menor sua nota. Peso: 3

- $\quad$ Rigidez: Trata-se de uma análise qualitativa da rigidez da máquina, mais tarde será feita uma análise quantitativa da solução escolhida. Peso: 2

- $\quad$ Peso: Trata-se da massa total da máquina para um mesmo volume de trabalho, leva em conta a quantidade de peças envolvidas e a volume ocupado pela máquina. Peso: 3

- $\quad$ Montagem: Trata-se da facilidade de montagem da máquina. Por se tratar de um projeto que será de tecnologia aberta, ela deve ser de fácil montagem. Peso: 2

B Matriz de decisão

Fazendo a análise qualitativa dos critérios acima citados, chegou-se na seguinte matriz de decisão:

Tabela I - Matriz de decisão. Fonte elaborada pelo autor.

\begin{tabular}{|c|c|c|c|}
\hline Critério & Peso & Portal & Bandeira \\
\hline Inércia Movida & 3,0 & 6,0 & 9,0 \\
\hline $\begin{array}{ll}\text { Vol. } & \text { De } \\
\text { Trabalho } & \end{array}$ & 2,0 & 9,0 & 10,0 \\
\hline Custo & 3,0 & 5,0 & 9,0 \\
\hline Rigidez & 2,0 & 10,0 & 7,0 \\
\hline Peso & 3,0 & 5,0 & 8,0 \\
\hline Montagem & 2,0 & 7,0 & 9,0 \\
\hline Total & & 100 & 130 \\
\hline
\end{tabular}

Tendo em vista estas considerações, a solução da estrutura escolhida foi a do tipo bandeira.

\subsection{Material extrudado}

Muitos materiais, como ABS (Acrilonitrila Butadieno Estireno) e policarbonato, necessitam que a plataforma onde o material é depositado seja aquecida. Garantindo assim a adesão do material. Além da temperatura, a aderência do material também depende da superfície. A cola do tipo bastão da $3 \mathrm{M}$ é usada para cobrir a superfície da plataforma e tem mostrado uma aderência adequada (HORVATH, 2014). Entretanto PLA não necessita que a plataforma seja aquecida, e nem necessita da cola bastão.

O PLA também é amplamente utilizado para impressões 3D, derivado do milho sendo assim biodegradável. O material permite sua extrusão a temperaturas mais baixas (entre 190 e 210 graus Celsius). Entretanto ele pode amolecer a temperaturas mais baixas também. Assim deve-se levar em conta esse fato quando utilizar este material (HORVARTH, 2014). Para que o PLA tenha uma boa aderência à mesa, é comum a utilização da Fita Crepe Profissional para pintura Scotch Blue da 3M.

\subsection{Solução final}

A solução escolhida foi uma estrutura do tipo bandeira, portanto com um eixo rotativo $\theta$, um eixo que se movimenta no raio R. Há um mancal em uma extremidade e com a outra extremidade em balanço. Há também um eixo que se movimenta no sentido vertical $Z$, com somente um motor de passo.

\subsection{Análise Mecânica}

A análise mecânica da estrutura foi feita considerando o modelo onde o eixo R é representado 
por duas barras cilíndricas engastadas. O pior caso deste modelo seria uma massa, correspondente à massa do bico aquecido e do suporte do bico, equivalente a aproximadamente $100 \mathrm{~g}$, na extremidade não suportada deste eixo. Foi considerado também o eixo $\mathrm{Z}$ como uma viga engastada.

O diagrama do corpo livre do eixo R está representado na Figura 4.
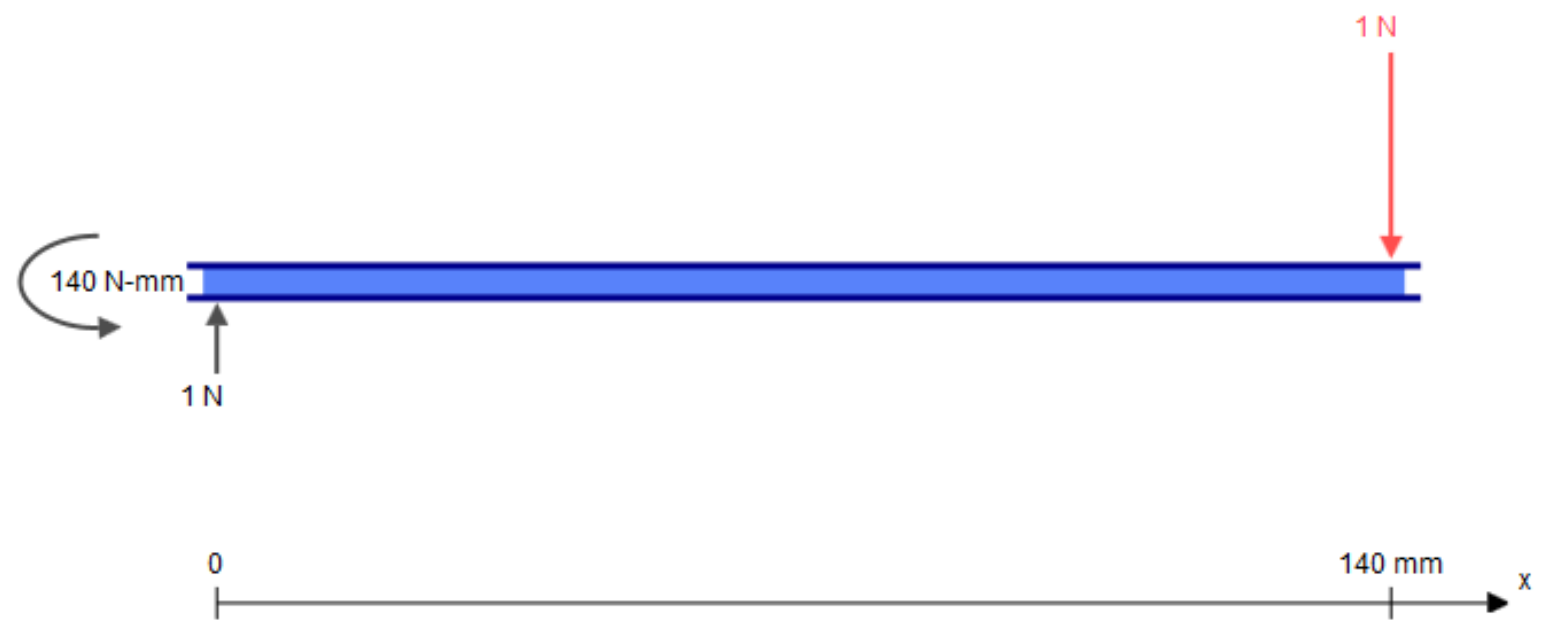

Figura 4 - Diagrama de corpo livre eixo R (Fonte: BEAM)

Fazendo a análise para uma só barra carregando metade da massa, tem-se que a deformação máxima da guia seria dada pela equação (1).

$\delta=\frac{F L^{3}}{3 E I}(1)$

Sendo que:

$I=\frac{\pi r^{4}}{4}(2)$

Substituindo os dados aplicados ao caso do eixo R de aço 1040 com 10mm de diâmetro temse que a máxima deformação é de aproximadamente $4,32 \mu \mathrm{m}$, abaixo do aceitável para este projeto.

O modelo que pode ser visto na Figura 5 que é regido pelas equações (3), (4), (5) e (6) (ENGINEERING, 2015).

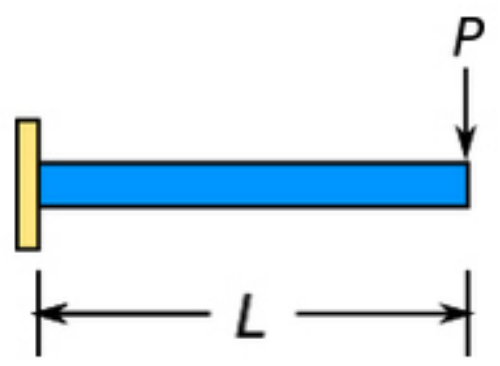

Figura 5 - Modelo para a simulação estática do eixo R (Fonte: ENGINEERING)

$\delta=\frac{P x^{2}}{6 E I}(3 L-x)(3)$

$\theta=\frac{P x}{2 E I}(2 L-x)(4)$

$M=P(x-L)(5)$

$V=P(6)$

Aplicando este modelo, dado que $\mathrm{L}=140 \mathrm{~mm}$, e P é a força em cada uma das duas barras no eixo $\mathrm{R}$, que supondo que seja igualmente equilibrada entre as duas, trata-se de aproximadamente 
$0,49 \mathrm{~N}$, temos que a maior deformação do eixo é de $4,42 \mu \mathrm{m}$, condizendo assim com a análise feita anteriormente.

Para a o eixo $\mathrm{Z}$, foi utilizado o modelo de uma viga engastada, com os esforços resultantes do eixo $R$ atuando nesta análise, este modelo pode ser visto na Figura 6

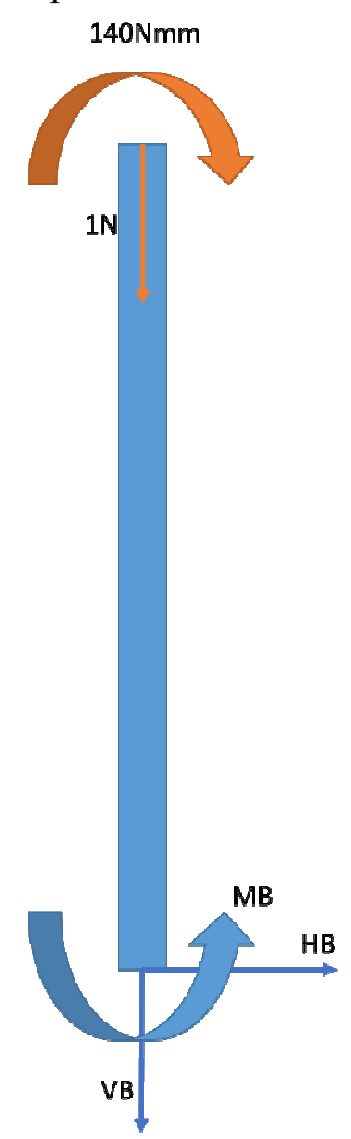

Figura 6 - Diagrama de corpo livre para a análise do eixo Z. Fonte elaborada pelo autor

Calculando as reações na base $B$ temos que:

- Reações horizontais nulas: $\mathrm{HB}=0$

- Reações verticais: $\mathrm{VB}=-1 \mathrm{~N}$

- Momento fletor: $\mathrm{MB}=140 \mathrm{Nmm}$

Com estas considerações, foi decidido que tanto o eixo $\mathrm{R}$ quanto o eixo $\mathrm{Z}$ seriam compostos de duas barras de $10 \mathrm{~mm}$ de diâmetro.

\subsection{Torque e Rotações}

Considerando-se desprezível a força de atrito com as guias, e a inexistência de forças de corte, de acordo com Adamowski (ADAMOWSKI, 2005)

$F_{t}=m \cdot \ddot{R}(7)$

Sendo Ft a força de tração e a derivada segunda de R como a aceleração do eixo R. Tem-se também que:

$T=r_{\text {polia }} \cdot F_{t}(8)$

Substituindo os valores corretamente, tem-se que o torque no motor é de aproximadamente $10 \mathrm{Nmm}$

Analogamente para o eixo $\mathrm{Z}, \mathrm{Fz}=4,44 \mathrm{~N}$, e para calcular o torque necessário, segundo Adamowski, tem-se as equações 9 e 10 :

$J_{P}=\left(m_{m}+m_{P}\right)\left(\frac{P}{2 \pi}\right)^{2}(9)$ 
$J_{f}=m f\left(\frac{d_{f}}{2}\right)^{2}(10)$

Utilizando-se um fuso M5 com passo de $0,8 \mathrm{~mm}$ temos que um avanço vertical de $5 \mathrm{~m} / \mathrm{s}$ equivale a $6250 \mathrm{rad} / \mathrm{s}$, sendo esta a condição máxima de trabalho.

Substituindo-se os valores tem-se que $J_{p}=1,187 \cdot 10^{-8} \mathrm{kgm}^{2}$ e $J_{f}=3,2 \cdot 10^{-6} \mathrm{kgm}^{2}$.A inércia conjugada é portanto a soma das duas e equivale a $J_{p}=3,21.10^{-6} \mathrm{kgm}^{2}$.

A inércia do motor de passo (NEMA 17) também deve ser levado em consideração, para isso verificamos o catálogo (PBCLINEAR, 2015) e chegamos a uma inércia do motor de $J_{r}=$ $5,7 \cdot 10^{-6} \mathrm{kgm}^{2}$. Aplicando à equação 11 do torque do motor tem-se que $T_{z}=35 \mathrm{Nmm}$.

$T_{z}=\alpha_{3} \cdot(J r+J c)$

Para o eixo $\theta$ definiu-se por exemplos anteriores uma relação de engrenamento de 10/36. Para que a velocidade seja compatível com o esperado, temos que para um raio de $5 \mathrm{~mm}$ a aceleração linear máxima é de $2 \mathrm{~m} / \mathrm{s}^{2}$. Isto corresponde a uma aceleração angular máxima de $400 \mathrm{rad} / \mathrm{s}^{2}$ pela equação 12.

$\theta_{M A X}^{\cdot .}=\frac{a}{r}$

Com a relação das engrenagens, isto corresponde a uma aceleração máxima no motor de passo $\left(\alpha_{\ddot{M A X}}\right)$ de $7776,47 \mathrm{rad} / \mathrm{s}^{2}$

Estimando a massa do disco que rotacional no eixo $\theta$ e sabendo que o momento de Inércia de um disco $I_{D}$ pode ser visto na equação (13). Portanto $I_{D}$ é $2,5.10-4 \mathrm{kgm}^{2}$.

$I_{D}=\frac{m r^{2}}{2}$

Sabe-se que o torque movido (Tmovido) é $0,1 \mathrm{Nm}$ pela equação 14 e o torque do motor (Tmotor) é $190 \mathrm{Nmm}$ pela equação 15.

$T_{\text {movido }}=\theta_{M A X} \cdot I_{D}(14)$

$T_{\text {motor }}=\frac{T_{\text {movido }}}{K_{1}}$

\subsection{Montagem e Construção}

Após definir todos as peças de acordo com os esforços e torques calculados na seção anterior, o projeto em CAD foi feito como pode ser visto na figura 7.

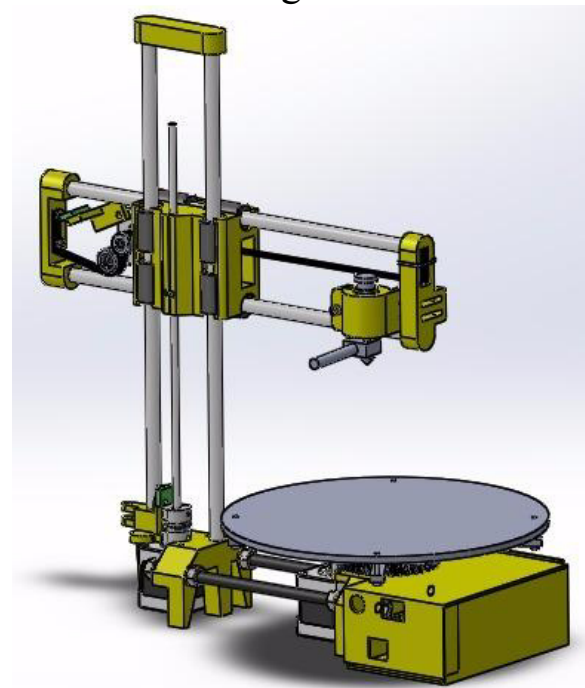

Figura 7 - Projeto final montado em CAD Solidworks. Fonte elaborada pelo autor.

A máquina montada pode ser vista na figura 8. A extrusora foi colocada na acoplada ao bico 
aquecido, devido a problemas com o cabo. Tanto a extrusora como o bico aquecido possuem extremidades de difícil acoplamento e a flexibilidade do cabo ocasiona na perda na transmissão do movimento do fio de PLA.

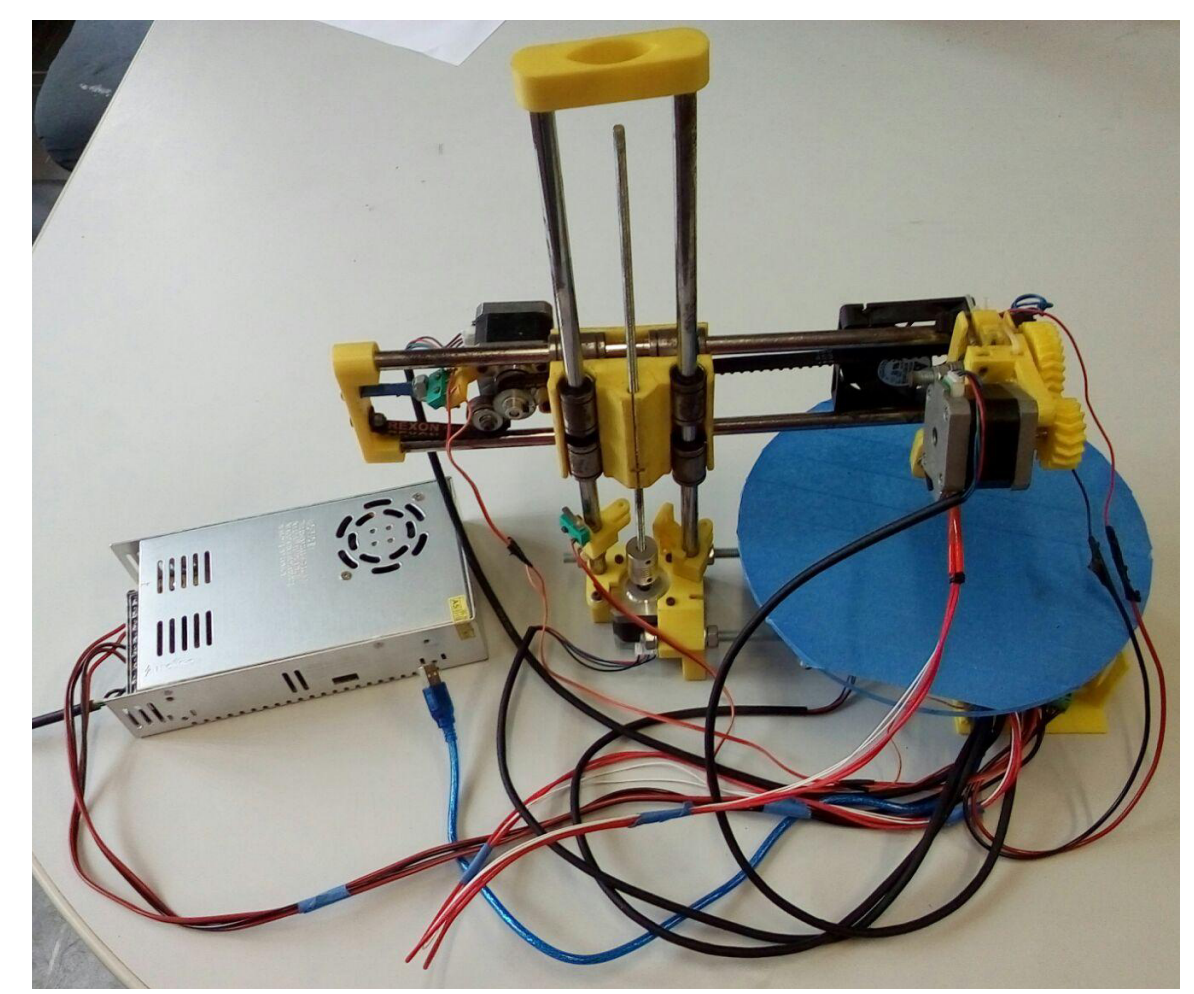

Figura 8 - Fotografia da Impressora 3D de coordenadas polares construída. Fonte elaborada pelo autor.

O gasto total com a construção da máquina foi de aproximadamente $\mathrm{R} \$ 1200,00$, bem abaixo das que são encontradas no mercado.

\subsection{Testes}

Com a máquina montada foi possível efetuar testes para verificar o desempenho da mesma. Os testes foram feitos em etapas demostradas a seguir

\section{A Movimentação manual}

Com auxílio do software Repetier-host, é possível movimentar cada eixo pelo jog, comando que possibilita selecionar quanto será movimentado a cada comando. Assim é possível comparar o com o deslocamento real. Este teste é feito com cada eixo, com jogs de 1 e $10 \mathrm{~mm}$.

\section{B Movimentação Automática}

Com o auxílio do Repetier-host e do Slic3er, peças testes de foram impressas para aferir se as dimensões impressas e comparar com o esperado. A peça de teste são anel de $\varnothing 10 \times 4 \mathrm{~mm}$, como mostrado na figura 9 . 


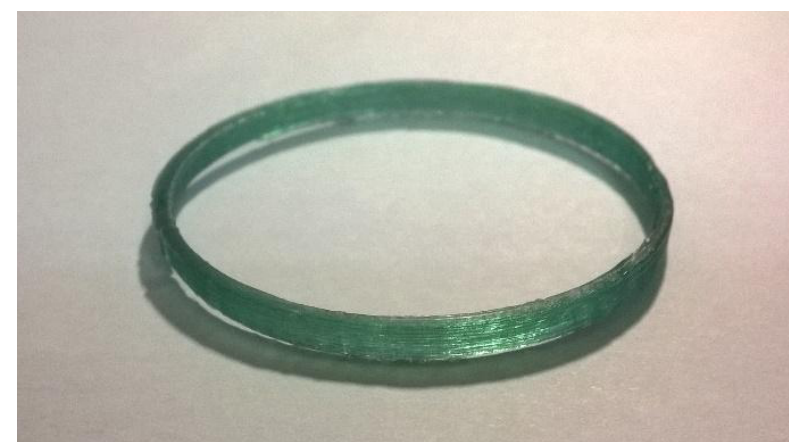

Figura 9 - Fotografia do anel impresso para testes. Fonte elaborada pelo autor.

\section{Conclusão}

Após o projeto e a construção da impressora 3D polar, concluiu-se que foi possível imprimir a peça teste com uma qualidade aceitável. Entretanto houve diversos problemas com o firmware, inclusive a conversão para polar não estava funcional e por este motivo foi feito manualmente.

A máquina ainda tem muito a ser melhorada. Por exemplo, a estrutura não é rígida o suficiente no eixo Z. Outro problema é a otimização para coordenadas polares que deve ser feita na conversão.

\section{REFERÊNCIAS BIBLIOGRÁFICAS}

ADAMOWSKI, J.C. Sistema de movimentação de uma máquina CNC - Controlador, drivers e motores. Disponível em <http://sites.poli.usp.br/d/PMR2450/aula4.pdf >. Acesso em 05 jun. 2015.

BEAM. Beam Calculator. Disponível em: $<$ http://bendingmomentdiagram.com/solve/>. Acesso em 15 jun. 2015.

ENGINEERING CALCULATOR- Beam Bending Calculator. Disponível em $<$ http://www.engineeringcalculator.net/beam_calculator.html $>$. Acesso em 15 jun. 2015.

HIRZEL, T. PWM. Disponível em $<$ https://www.arduino.cc/en/Tutorial/PWM>. Acesso em: 20 out. 2015.

HORVATH, J.C. Mastering 3D Printing: Modeling, Printing, and Prototyping with RepRap-Style 3D Printers, Berkley: Apress. 2014. ISBN 978-1-4842-0026-1

MAJCHER. How to Build 3-D Printing. Disponível em

$<$ http://www.technologyreview.com/news/530721/how-to-build-3-d-printing/> Acesso em: 19 nov. 2015.

PBCLINEAR. Stepper Motor Support Document. Disponível em:

$<$ http://www.pbclinear.com/Download/DataSheet/Stepper-Motor-Support-Document.pdf $>$. Acesso em: 05 jun. 2015.

REPRAP. RepRap. RAMPS 1.4. 20 fev. 2015. Disponível em

$<$ http://reprap.org/wiki/RAMPS_1.4>. Acesso em: 01 abr. 2015.

\section{Title: Development of a polar coordinates 3D Printer \{English title\}}

Abstract - With the growing tendency of product customization, and with more and more complexes geometries, 3D printers provide great versatility and have been used more often for all kinds of use, be it professional use or personal use. With a manufacturing process called additive manufacturing, 3D printers become popular to several users. With the intention to make this technology even more accessible for the community, it was developed a polar coordinates $3 \mathrm{~d}$ printer, with the advantage of 
having fewer components and printed parts in its constitution compared to the traditional Cartesians 3D Printers.

Keywords -3D Printer, RepRap, Polar Coordinates

\section{Kalenin de Moraes Branco}

Aluno de Engenharia Mecatrônica da Escola Politécnica da Universidade de São Paulo.

E-mail: kalenin.poli@gmail.com

\section{Mariana Wong Yoshikawa}

Aluna de Engenharia Mecatrônica da Escola Politécnica da Universidade de São Paulo.

E-mail: yoshikawa.mariana@gmail.com 